\title{
Transfer reactions on light exotic nuclei studied with CHIMERA detector at LNS
}

Giuseppe Cardella ${ }^{1 \mathrm{a}}$, Luis Acosta ${ }^{1,9}$, Francesca Amorini ${ }^{2}$, Lucrezia Auditore $^{4}$, Ionela Berceanu ${ }^{7}$, Mihir Chatterjiee $^{8}$, Enrico DeFilippo ${ }^{1}$, Laura Francalanza ${ }^{2,3}$, Rita Gianì ${ }^{2,3}$, Laura Grassi ${ }^{1}$, Elena La Guidara ${ }^{1}$, Gaetano Lanzalone ${ }^{2,5}$, Ivano Lombardo ${ }^{6}$, Dario Loria ${ }^{4}$, Triestino Minniti ${ }^{4}$, Angelo Pagano ${ }^{1}$, Emanuele V. Pagano ${ }^{2,3}$, Massimo Papa ${ }^{1}$, Sara Pirrone ${ }^{1}$, Giuseppe Politi ${ }^{1,3}$, Amalia Pop ${ }^{7}$, Francesco Porto ${ }^{2,3}$, Francesca Rizzo $^{2,3}$, Elio Rosato ${ }^{6}$, Paolo Russotto ${ }^{1}$, Simone Santoro ${ }^{4}$, Antonio Trifirò ${ }^{4}$, Marina Trimarchi $^{4}$, Giuseppe Verde ${ }^{1}$, Mariano Vigilante ${ }^{6}$

${ }^{1}$ INFN - Sezione di Catania, Via S. Sofia 64, 95123 Catania, Italy

${ }^{2}$ INFN - Laboratori Nazionali del Sud, Via S. Sofia 44, Catania, Italy

${ }^{3}$ Dip. di Fisica e Astronomia, Università di Catania, Via S. Sofia 64, Catania, Italy

${ }^{4}$ INFN Gruppo collegato di Messina \& Dip. di Fisica, Università di Messina, Italy

${ }^{5}$ Facoltà di Ingegneria e Architettura, Università Kore, Enna, Italy

${ }^{6}$ INFN Sezione di Napoli \& Dipartimento di Fisica, Università Federico II, Napoli, Italy

${ }^{7}$ Institute for Physics and Nuclear Engineering, Bucharest, Romania

${ }^{8}$ Saha Institute for Nuclear Physics, Kolkata, India

${ }^{9}$ Departamento de Fisica Aplicada, Universidad de Huelva, Huelva, Spain

\begin{abstract}
The kinematical coincidence method is used to extract angular distribution of elastic scattering and transfer reactions. The detected light particle energy spectra are used to extract the angular distribution with around $1^{\circ}$ resolution in the Center of Mass (CM) system. Examples with ${ }^{10} \mathrm{Be}$ beam are presented. In the case of proton scattering, $\gamma$-ray coincidences are used to discriminate excited levels population from elastic scattering.
\end{abstract}

\section{Introduction}

Relatively large yields of various neutron rich exotic beams $\left({ }^{6} \mathrm{He},{ }^{8,9} \mathrm{Li},{ }^{10,11} \mathrm{Be},{ }^{13} \mathrm{~B},{ }^{16,17} \mathrm{C}\right)$, produced through in-flight fragmentation of ${ }^{18} \mathrm{O}$ beams at $55 \mathrm{~A} \cdot \mathrm{MeV}$, are available at LNS [1]. Using the CHIMERA detector [2,3], we started a campaign to study transfer reactions, with proton and deuteron enriched targets. The kinematical coincidence method was used to extract high resolution angular distributions of binary reactions from the measured light particle energy spectra [4]. We were able to

\footnotetext{
${ }^{\mathrm{a}}$ Corresponding author: cardella@ct.infn.it
} 
disentangle some excited levels, using $\gamma$-rays detected in the CsI(Tl) detectors of the Chimera telescopes. The complete analysis of these data will be part of a systematic research for a dependence of cross sections by the observed or claimed halo structures of light neutron rich nuclei.

\section{Experimental setup and data analysis}

The fragmentation beam was produced by using an ${ }^{18} \mathrm{O}^{7+}$ primary beam delivered by the INFN-LNS Superconducting Cyclotron at $55 \mathrm{~A} \cdot \mathrm{MeV}$. The beam line magnetic elements were set in order to maximize production of ${ }^{11} \mathrm{Be}$ ions $(\mathrm{B} \rho=2.78 \mathrm{Tm})$.

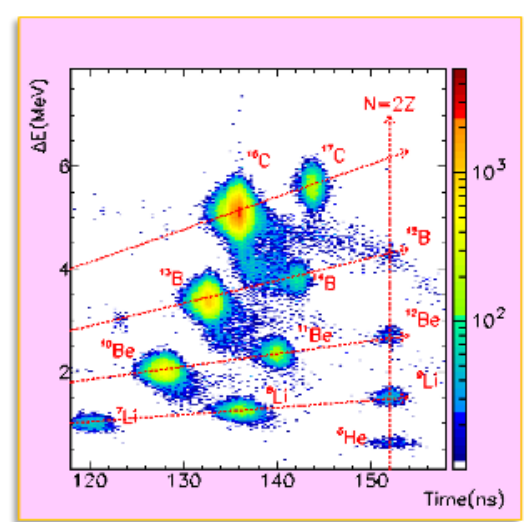

Figure 1. Identification scatter plot of the fragmentation beam. Arrows show the loci for the different charges and $\mathrm{N} / \mathrm{Z}$ ratio.

The beam was identified particle by particle by using a tagging system consisting of a Double Side Silicon Strip Detector (DSSSD) and a large surface Micro-Channel Plate (MCP) [5]. The MCP detector was placed approximately $13 \mathrm{~m}$ before the tagging strip and it was used as start of the time of flight (TOF) measurement of the beam particles ( the stop being delivered by the DSSSD ). In fig. 1 it is reported the quality of the identification obtained by plotting the TOF as a function of the energy loss $(\Delta \mathrm{E})$ measured in the DSSSD.

The first step of the data analysis consists on the selection of the beam under study with the use of appropriate cuts in the scatter plot already shown in fig.1. Events were further selected, searching for kinematical coincidences. For this reason only events with charged particles multiplicity equal to two were analyzed requiring also a total detected charge equal to the beam plus target charge. This was very useful to strongly reduce contamination from carbon in the plastic target, and from reactions in the DSSSD. Other constraints were taken into account by using conservation laws.

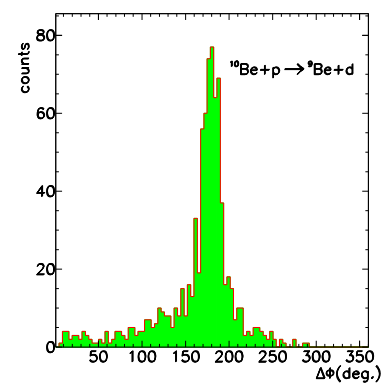

Figure 2. Relative azimuthal angle $\Delta \phi$ measured for coincidence events in the reaction ${ }^{10} \mathrm{Be}+\mathrm{p} \rightarrow{ }^{9} \mathrm{Be}+\mathrm{d}$ 
Firstly, due to momentum conservation the relative azimuthal angle $\Delta \phi$ between the two fragments must be $180^{\circ}$. In fig. 2, we plot this angle as measured for the reactions, ${ }^{10} \mathrm{Be}+\mathrm{p} \rightarrow{ }^{9} \mathrm{Be}+\mathrm{d}$. We can recognize the binary events concentrated in the peak around $180^{\circ}$. A further selection, based on energy conservation law, requires that the sum of kinetic energies of the two detected particles is equal to the beam energy plus the Q-value.

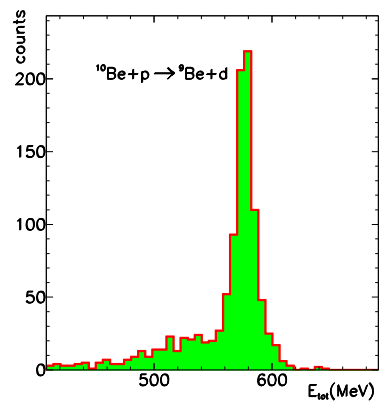

Figure 3. Total energy spectrum for the reaction ${ }^{10} \mathrm{Be}+\mathrm{p} \rightarrow{ }^{9} \mathrm{Be}+\mathrm{d}$

In fig. 3 we plot the total energy detected in coincidences between deuterons and $\mathrm{Be}$ ions in the reaction ${ }^{10} \mathrm{Be}+\mathrm{p}$. The events fulfilling the energy conservation selection can be seen close to the value of $580 \mathrm{MeV}$, roughly corresponding to the total available energy. Due to the relatively poor CsI(Tl) energy resolution for heavy fragments, this total energy peak is rather large (FWHM $\sim 20 \mathrm{MeV}$ ) so it is difficult, in principle, to discriminate the decay path towards the ground state. However, in this case, only ${ }^{9} \mathrm{Be}_{\mathrm{gs}}$ can be populated, because the first ${ }^{9} \mathrm{Be}$ excited level, at $1.684 \mathrm{MeV}$, is unbound, decaying to the $n+2 \alpha$ channel. Being the final channel well defined, we can simply convert the deuteron energy spectrum shown in fig.4a, in the Center of Mass (CM) angular distribution. This can be done by using kinematic relations, taking into account beam intensity, target thickness, and detector efficiency [6].

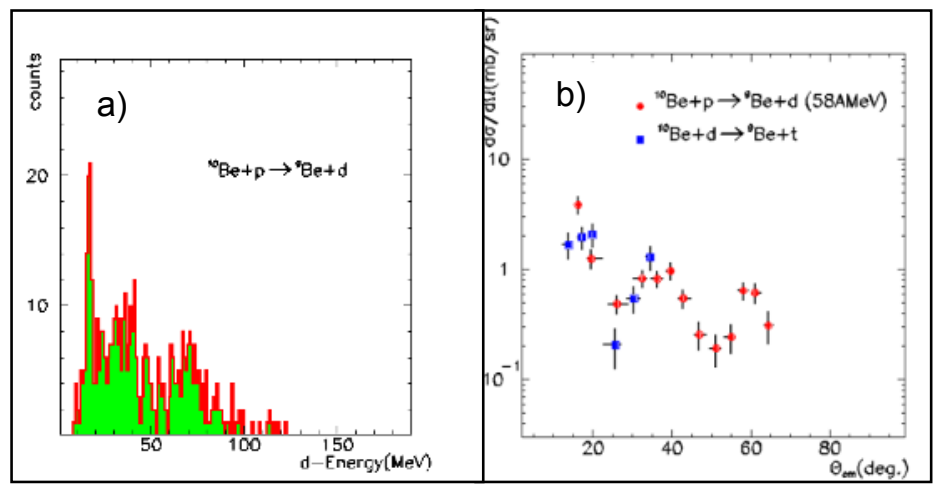

Figure 4. a) Deuteron energy spectrum from kinematical coincidences and b) extracted angular distribution in the reaction ${ }^{10} \mathrm{Be}+\mathrm{p} \rightarrow{ }^{9} \mathrm{Be}+\mathrm{d}$ (full dots). In b) also the angular distribution from the ${ }^{10} \mathrm{Be}+\mathrm{d} \rightarrow{ }^{9} \mathrm{Be}+\mathrm{t}$ reaction is plotted (full square).

In fig. $4 \mathrm{~b}$ the obtained angular distribution for the $p, d$ (dots) and $d, t$ reactions (squares) are shown. The cross sections and the angular distributions for the two reaction channels are quite similar. It seems that the difference in the final state can be neglected and that the cross section is determined mainly by the characteristics of the transferred neutron. The value of the size of the angular bins of each point was chosen by the need to get a reasonable statistical error. The angular resolution evaluated from the energy resolution measured with proton elastic scattering is of the order of $1^{\circ}$. We emphasize that this method to extract the angular distribution allows to automatically correct for the position and direction spread of fragmentation radioactive beams. 
As above stated the total energy resolution is not enough to discriminate excited levels. The angular distribution obtained for the elastic channel of ${ }^{10} \mathrm{Be}+\mathrm{p}$ reaction, shown as full dots in fig. 5a, is affected by this problem. We note a change of slope around $40^{\circ}$, not observed in previous data, measured with SPEG [7], shown as open dots. We cannot disentangle the contribution of bound excited levels of ${ }^{10} \mathrm{Be}$. However such levels decay by $\gamma$-ray emission. The second stage of a CHIMERA telescope is a large $\mathrm{CsI}(\mathrm{Tl})$ detector with photodiode readout, and we are able to well identify $\gamma$-rays [8]. In fig. 5b we shown the $\gamma$-ray spectrum detected in coincidence with protons in such reaction. Even if the resolution is scarce and, due to some electronic thresholds, the efficiency was low in this experiment, the spectrum is what expected from the decay of excited ${ }^{10} \mathrm{Be}$ levels. The angular distribution of protons tagged with such $\gamma$-rays well explains the change of slope observed around $40^{\circ}$ (full triangles in fig.5a).

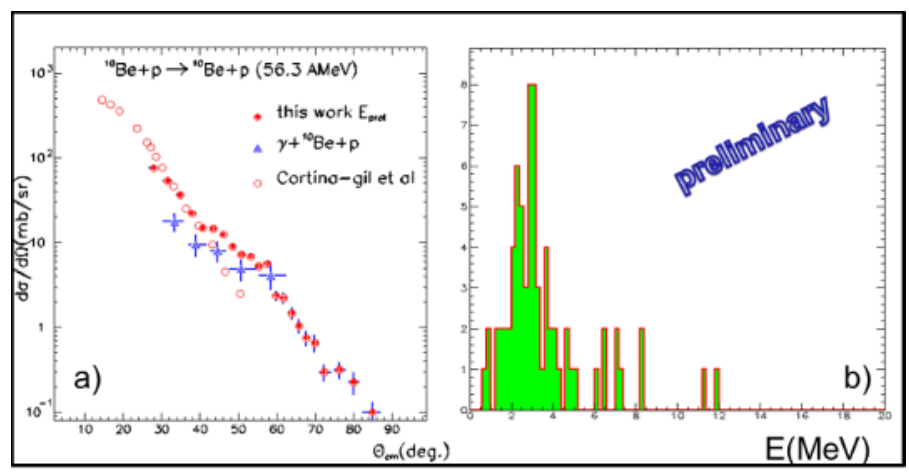

Figure 5. a) Angular distribution measured for the proton scattering in ${ }^{10} \mathrm{Be}+\mathrm{p}$ reaction (full dots), compared to the data of ref. [7] (open dots). The angular distribution of protons detected in coincidence with $\gamma$-rays (see b), is also plotted (triangles). b) $\gamma$-rays energy spectrum.

\section{Conclusions and outlook}

The power of the kinematical coincidence method was shown. This method is particularly suitable for a $4 \pi$ detector as CHIMERA, where kinematical coincidences are automatically recorded. The availability of high efficiency $\gamma$-ray detectors, as the CsI(Tl) of the apparatus, can further help in the selection of the final channel. Future measurements will also profit of the availability of the new FARCOS prototype array [9] that can enhance the CHIMERA performances for the detection of both heavy residue and light fragments depending on the experimental needs.

\section{References}

1. $\quad$ see http://fribs.lns.infn.it/upgrade-results.html

2. A.Pagano et al, Nucl. Phys. A 734, 504 (2004)

3. A.Pagano, Nuclear Physics News, 22:1, 25(2012)

4. E.De Filippo et al Phys. Rev. C 71, 044602 (2005)

5. I.Lombardo et al Nucl. Phys. B 215, 272 (2011)

6. L.Acosta et al NIM A 715, 56 (2013)

7. M.D.Cortina Gil et al Phys.Lett.b 401, 9 (1997)

8. M.Alderighi et al NIM A 489, 257 (2002) 257.

9. L. Acosta et al., EPJ Web of Conferences, 31 ,0035 (2012). 